\title{
Examination of Exercise-Induced Skeletal and Cardiac Muscle Damage in Terms of Smoking
}

\author{
Gokhan Ipekoglu ${ }^{1}$, Halil Taskın², Omer Senel ${ }^{3}$
}

Affiliations: 'Sinop University, Sports Sciences Faculty, Sinop, Turkey, ${ }^{2}$ Selcuk University, Sports Sciences, Faculty, Konya, Turkey, ${ }^{3}$ Gazi University, Sports Sciences Faculty, Ankara, Turkey

Correspondence: G. Ipekoglu, Sinop University, Sports Sciences Faculty, Korucuk Koyu, Trafo Mah. No: 36, 57000, Sinop, Turkey, e-mail: gipekoglu@sinop.edu.tr

ABSTRACT This study aimed to investigate the effect of acute endurance exercise on cardiac and skeletal muscles in smokers and non-smokers. Eight daily smokers (28.44 \pm 3.94 years) and nine non-smokers (29.62 \pm 3.46 years) were included. The subjects were not trained and performed continuous endurance exercise on a treadmill for 40 minutes at $70 \%$ of maximal heart rate. Venous blood samples were collected at baseline [preexercise (PRE)], at immediately after the exercise [post-exercise (POST)], at 2 hours after the exercise (2h), at 24 hours after the exercise $(24 \mathrm{~h})$ to measure lactate dehydrogenase (LDH), creatine kinase(CK), creatine kinase-myocardial band (CK-MB), cardiac troponin T (TN-T), and myoglobin levels. A progressive increase was observed in all exercise-induced muscle damage parameters of the smoker and non-smoker from PRE to $2 \mathrm{~h}$. CK, myoglobin and T-NT levels of smokers were significantly higher than non-smokers at $24 \mathrm{~h}(\mathrm{p}=0.039$, $\mathrm{p}=0.018 \mathrm{p}=0.008$, respectively). No significant difference was found between the smoking and non-smoking groups at all time points regarding $\mathrm{CK}-\mathrm{MB}$ and LDH levels $(\mathrm{p}>0.05)$. Acute endurance exercise leads to more skeletal and myocardial damage in smokers compared to non-smokers. Smoking may increase the risk of cardiovascular events during both exercise and daily physical activity.

KEY WORDS smoke, cardiac damage, muscle damage, aerobic exercise

@MJSSMontenegro

SKELETAL AND CARDIAC MUSCLE DAMAGE IN TERMS OF SMOKING

http://mjssm.me/?sekcija=article\&artid=176

\section{Introduction}

The long-term adverse effects of cigarette smoking on human health have been proven by experts. It is known that smokers have a higher risk of death, heart diseases, and cancer (Srivastava et al., 2000). In the year 2000, an estimated 1.62 million cardiovascular deaths occurred due to smoking in the world; and this figure constitutes $11 \%$ of the total global cardiovascular deaths (Ezzati et al., 2005). Increasing physical activity is a potential strategy that can reduce the harmful effects of chronic smoking. However, it is unknown whether regular exercise alleviates the adverse effects of smoking on large artery distensibility (Tanaka \& Safar, 2005; Park et al., 2014). Skeletal muscle performance may be affected negatively in smokers since oxygen extraction is limited in smokers than non-smokers (King et al., 1987). Although muscular damage is closely related to the intensity of the exercise, those who are unaccustomed to exercise can frequently cause muscular damage (Borg, 1982; Brown \& Hill, 1991; Bryne \& Eston, 2002; Colakoglu et al., 2014).

The affinity of haemoglobin for CO is 200 to 250 times greater than its affinity for oxygen. This results in competitive inhibition of oxygen release due to a shift in the oxygen-haemoglobin dissociation curve, reduced oxygen delivery, and subsequent tissue hypoxia (Ernst \& Zibak, 1998; Fox et al., 1993). Smoking by causing catecholamine stimulation may lead to decreased maximal oxygen utilization, deterioration of the respiratory system and long-term cardiac damage (Green et al., 1986; Powders et al., 1989; Laustiola et al., 1988). Since the decrease in oxygen utilization capacity will also affect the amount of oxygen delivered to the tissues, the damage and recovery in muscle tissues will be expected to occur subsequently.

An increase in creatine kinase (CK) and lactate dehydrogenase (LDH), as indices of cellular necrosis and tissue damage in skeletal muscles, is widely used in the diagnosis of skeletal muscle diseases (Brancaccio et al., 2006). 
The diagnosis of myocardial injury is aided by a number of biomarker assays including CK isoenzyme MB mass (CK-MB), cardiac troponin T (cTnT) and myoglobin (MYB) (Hachey et al., 2016; Nie et al., 2011).

Although results from previous studies are somewhat conflicting, it is usually agreed that cigarette smoking will reduce the capacity of an individual to perform aerobic exercise (Morton \& Holmik, 1985). An expanded body of literature mentions that aerobic exercise has positive effects on physical functioning, psychological states, and mental processes (Martinsen \& Stephens, 1994). Moreover, many of the variables influenced by regular exercise are the same ones that are negatively affected by smoking withdrawal (Prapavessis et al., 2007).

Although the adverse effects of smoking have been studied, the extent to which smoking damages the heart and skeletal muscle in relation to exercise types is an issue of concern. For this reason, this study aimed to examine the changes in cardiac and skeletal muscle injury levels in acute endurance exercise between the smoking and non-smoking individuals.

\section{Methods}

Participants

The study was carried out on eight male smokers (28.44 \pm 3.94 years), who smoked at least 15 cigarettes per day for five years, and on nine male non-smokers (29.62 \pm 3.46 years) (Table 1). All subjects were untrained. Subjects with any diseases (hypertension, thyroid, diabetes, cardiac, etc.) were not included in this study. We informed the participants in detail about the objectives of the study according to the Helsinki Declaration and obtained informed consent. This study was approved by the Ethics Committee of the University (decision number 40990478-05.99).

\begin{tabular}{ccc} 
TABLE 1 The physical properties of smoking and non-smoking groups. & $\begin{array}{c}\text { Smoker } \\
(\mathbf{n = 9 )}\end{array}$ & $\begin{array}{c}\text { Non-smoker } \\
(\mathbf{n = 8})\end{array}$ \\
\hline Age (years) & $28.44 \pm 3.94$ & $29.62 \pm 3.46$ \\
Body Height (cm) & $175.45 \pm 4.70$ & $173.87 \pm 3.96$ \\
Body Weight $(\mathrm{kg})$ & $73.11 \pm 6.95$ & $71.12 \pm 5.61$ \\
BMI (kg/m2) & $23.17 \pm 1.96$ & $22.72 \pm 2.72$ \\
\hline
\end{tabular}

Note. Mean \pm SD *Significant difference between groups $(p<0.05)$.

\section{Exercise protocol}

Subjects performed continuous endurance exercise on a treadmill (Dunlop EL900) for 40 minutes at 70\% of maximal heart rate (HR). The target HR range was calculated as $0.7 \times$ (peak HR - resting HR) + (resting HR), in accordance with the method of Karvonen. To verify and record the duration and intensity of exercise, participants wore a heart rate monitor (Polar RS400, Polar, Kempele, Finland).

\section{Measurements}

A total of $15 \mathrm{cc}$ venous blood samples were extracted from the forearm pre-exercise (PRE), post-exercise (POST), post-exercise 2 hours ( $2 \mathrm{~h}$ ), post-exercise 24 hours (24h) to measure levels of TN-T, MYB, CK, CK$\mathrm{MB}$, and $\mathrm{LDH}$. The blood samples were first centrifuged at a rate of 5000 revolution/minute, and the upper phases were transferred to Eppendorf tubes and kept at $-80^{\circ} \mathrm{C}$ until the use. The concentrations were studied with the Beckman Coulter method using a Beckman Coulter AU2700 Plus biochemical auto-analyser with Beckman Coulter kits (Colakoglu et al., 2016).

\section{Statistics analysis}

The data were analysed with the SPSS version 22 software package, and the distribution of the analysed variables was assessed using a Shapiro-Wilk test. The results showed that the distributions deviated from the normal distribution. The biochemical data were non-parametrically distributed and were expressed as the median (interquartile range) (IQR). The Mann-Whitney $\mathrm{U}$ test was used to compare the PRE, POST, $2 \mathrm{~h}$, and $24 \mathrm{~h}$ values between the two groups. A Friedman rank test was undertaken to evaluate the statistical differences in time for each parameter. When a significant F-value in Friedmans' analysis was found, a post-hoc test was used to determine the between-means differences. The level of significance was set to be $\mathrm{p}<0.05$.

\section{Results}

According to the results, the TN-T levels of the non-smokers showed no significant changes at all time points ( $\mathrm{p}>0.05)$ except for $24 \mathrm{~h}(\mathrm{p}=0.008)$ when compared with pre-exercise. The $24 \mathrm{~h}$ TN-T level was significantly lower than PRE in non-smokers ( $\mathrm{p}=0.007)$. Compared with pre-exercise, the TN-T activity of the smoking group was significantly elevated at post-exercise $(\mathrm{p}=0.040)$. Furthermore, there were no significant differences among the $2 \mathrm{~h}$ and $24 \mathrm{~h}$ TN-T levels of the smoking group ( $\mathrm{p}=0.641$ ) (Table 2 ). 
TABLE 2 Changes in the serum troponin (pg/ml), myoglobin $(\mathrm{ng} / \mathrm{ml}), \mathrm{CK}(\mathrm{u} / \mathrm{l}), \mathrm{CK}-\mathrm{MB}(\mathrm{u} / \mathrm{l})$ and LDH $(\mathrm{u} / \mathrm{l})$ concentrations at different time points

\begin{tabular}{|c|c|c|c|c|c|}
\hline Variables & Groups & Pre & Post & $2 \mathrm{hr}$ & $24 \mathrm{hr}$ \\
\hline \multirow{2}{*}{ Troponin (pg/ml) } & Smoker & $5.17(4.32-6.84)$ & $5.44(3.00-8.87)^{*}$ & $5.79(3.00-18.37)$ & $4.82(3.00-6.10)$ \\
\hline & Non-smoker & $5.75(4.78-6.99)$ & $5.84(4.00-7.52)$ & $8.86(3.00-22.26)$ & $3.00(3.00-5.60)^{*}$ \\
\hline \multirow{2}{*}{ Myoglobin (ng/ml) } & Smoker & $40.07(23.01-226)$ & $54.52(23.58-234)$ & 88.40 (47.95-291.3) & $49.76(28.94-159.1)$ \\
\hline & Non-smoker & $29.85(21-70.20)$ & $50.51(33.31-110.4)^{*}$ & $58.28(39.75-188.20)$ & $35.06(26.47-219.1)$ \\
\hline \multirow{2}{*}{ CK (u/l) } & Smoker & $116.00(47-996)$ & $150.50(55-1056)^{*}$ & $149.50(72-1073)^{*}$ & $275.50(103-938)^{*}$ \\
\hline & Non-smoker & $132.00(90-316)$ & $157.00(107-383)^{*}$ & $181.00(128-361)^{*}$ & $235.00(133-802)^{*}$ \\
\hline \multirow{2}{*}{ CK-MB (u/l) } & Smoker & $15.50(11-26)$ & $17.50(12-30)^{*}$ & $16.00(11-26)$ & $16.00(11-24)$ \\
\hline & Non-smoker & 15.00 (13-19) & $18.00(17-23)^{*}$ & $16.00(14-23)^{*}$ & $17.00(13-44)$ \\
\hline \multirow{2}{*}{$\mathrm{LDH}(\mathrm{u} / \mathrm{l})$} & Smoker & $136.50(116-228)$ & $179.00(144-242)^{*}$ & $166.00(135-262)^{*}$ & $168.50(108-251)$ \\
\hline & Non-smoker & $161.00(92-195)$ & $177.00(144-228)^{*}$ & $196.00(120-228)^{*}$ & $188.11(110-357)$ \\
\hline
\end{tabular}

Note. Median (Interquartile Range); ${ }^{*}$ Significant difference compared with Pre $(p<0.05)$.

The TN-T levels were significantly different between the smoking and non-smoking groups at PRE $(\mathrm{p}=0.039)$ and $24 \mathrm{~h}(\mathrm{p}=0.008)$ (Figure 1$)$.

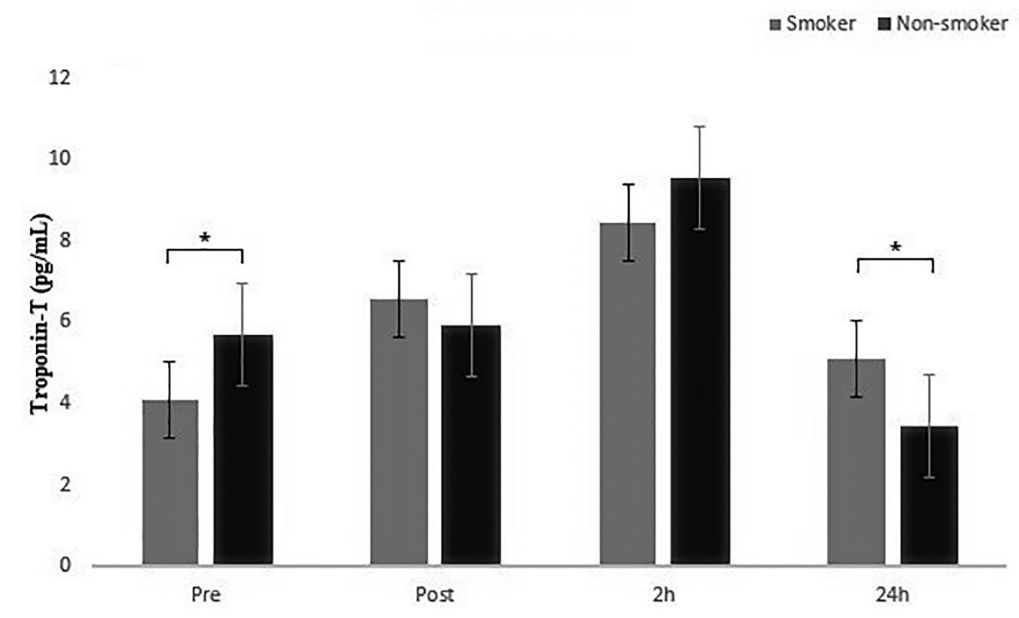

FIGURE 1 Changes in the serum cardiac troponin-T (pg/mL). ${ }^{*} \mathrm{p}<0.05$ indicates significant difference smoker vs. non-smoker.

The MYB levels of the non-smokers showed no significant changes at all time points ( $p>0.05)$, except for POST ( $\mathrm{p}=0.015)$, when compared with pre-exercise. The MYB activity of the smoking group significantly increased only at $2 \mathrm{~h}(\mathrm{p}=0.045)$. Although the PRE MYB levels were similar between the groups $(\mathrm{p}=0.137)$, the POST ( $\mathrm{p}=0.027), 2 \mathrm{~h}(\mathrm{p}=0.043)$, and $24 \mathrm{~h}(\mathrm{p}=0.039)$ MYB levels were significantly higher in the smoking group compared to non-smoking group (Figure 2 ).

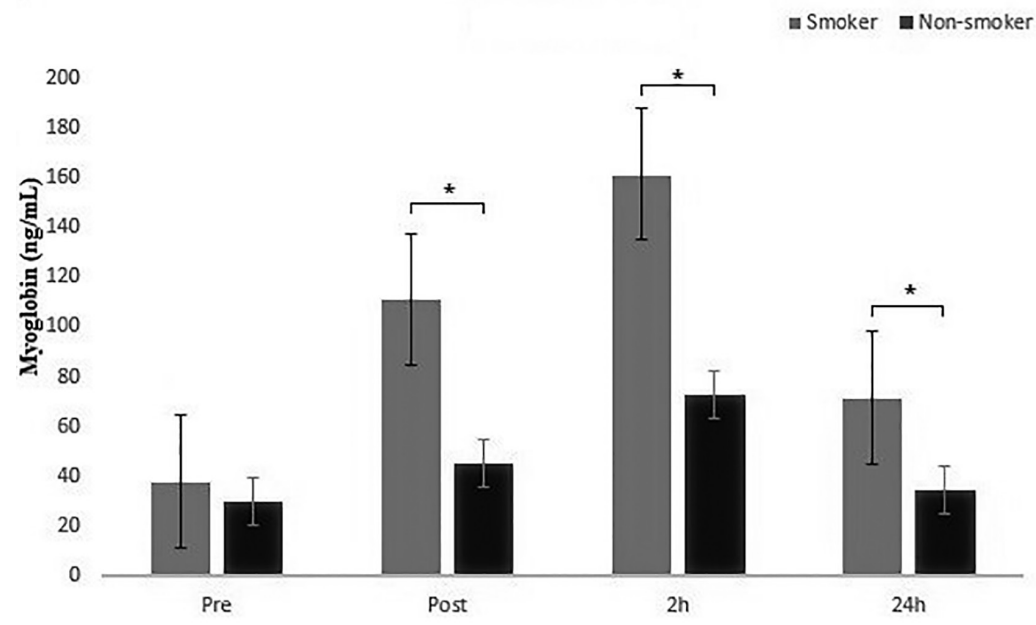

FIGURE 2 Changes in the serum cardiac troponin-T (pg/mL). ${ }^{*} p<0.05$ indicates significant difference smoker vs. non-smoker. 
The results indicated significant difference between CK activities of the smoking group. It increased significantly at post-exercise $(\mathrm{p}=0.008), 2 \mathrm{~h}(\mathrm{p}=0.031)$ and $24 \mathrm{~h}(\mathrm{p}=0.040)$. Furthermore, the CK activities of the non-smoking group were elevated at post-exercise $(\mathrm{p}=0.000), 2 \mathrm{~h}(\mathrm{p}=0.011)$, and $24 \mathrm{~h}(\mathrm{p}=0.008)$ (Table 2). The $\mathrm{CK}$ level was significantly higher in the smoking group than the non-smoking group at $24 \mathrm{~h}(\mathrm{p}=0.018)$. There was no significant difference between the groups at other time points $(\mathrm{p}>0.05)$ (Figure 3 ).

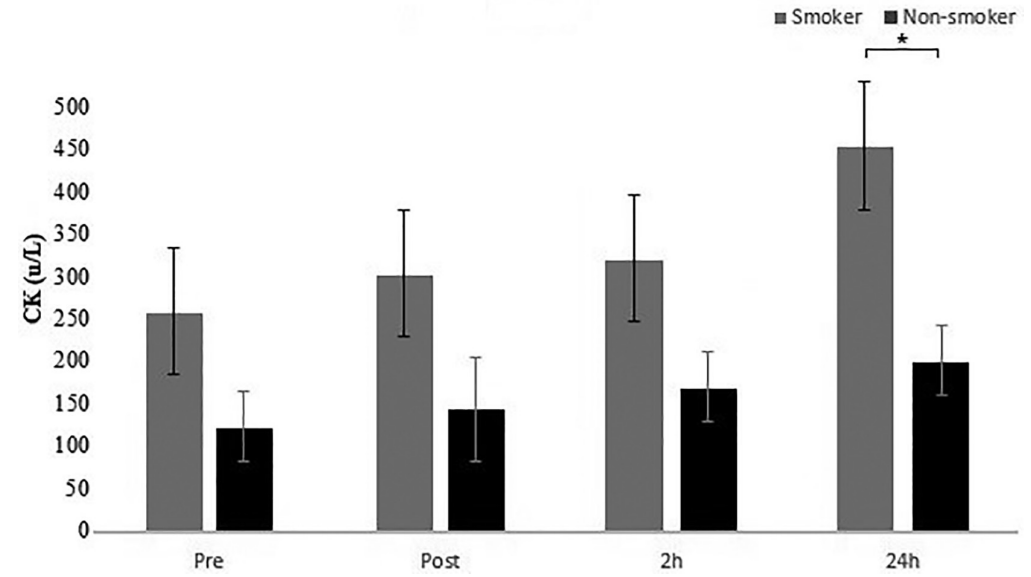

FIGURE 3 Changes in the serum creatine kinase(u/L). * $p<0.05$ indicates significant difference smoker vs. nonsmoker.

Post hoc analysis revealed that the $2 \mathrm{~h}$ CK-MB level for the non-smoking group was higher than both PRE and POST ( $p=0.000, p=0.007$, respectively). Furthermore, the CK-MB activity of the smoking group significantly increased only at POST $(\mathrm{p}=0.045)$. No significant difference was found between the smoking and non-smoking groups at all time points regarding CK-MB levels ( $\mathrm{p}>0.05)$ (Figure 4).

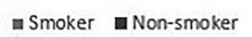

25

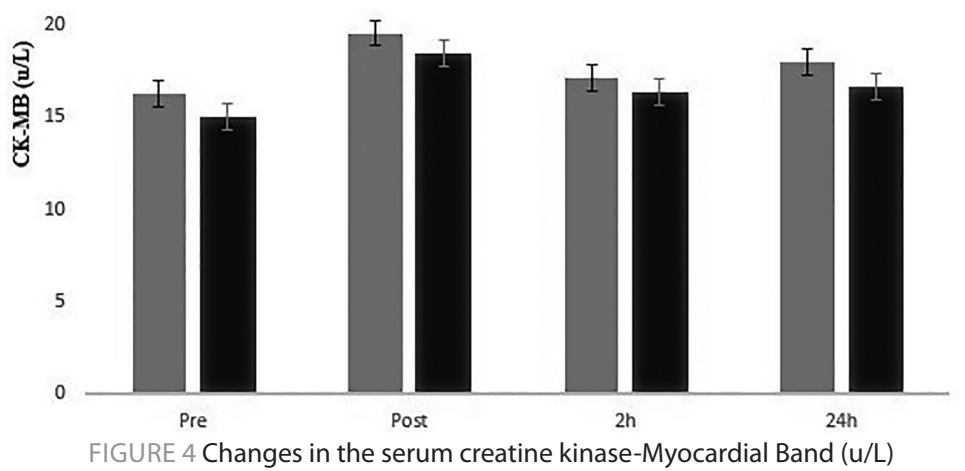

In both groups, both post-exercise LDH levels ( $\mathrm{n}$-smoker $\mathrm{p}=0.001$, smoker $\mathrm{p}=0.012$ ) and LDH levels 2 hours after exercise ( $n$-smoker $p=0.000$, smoker $p=0.042$ ) showed a significant increase compared to the pre-exercise LDH levels. No significant difference was found between the smoking and non-smoking groups at all time points regarding LDH levels ( $\mathrm{p}>0.05$ ) (Figure 5).

- Smoker anon-smoker

250

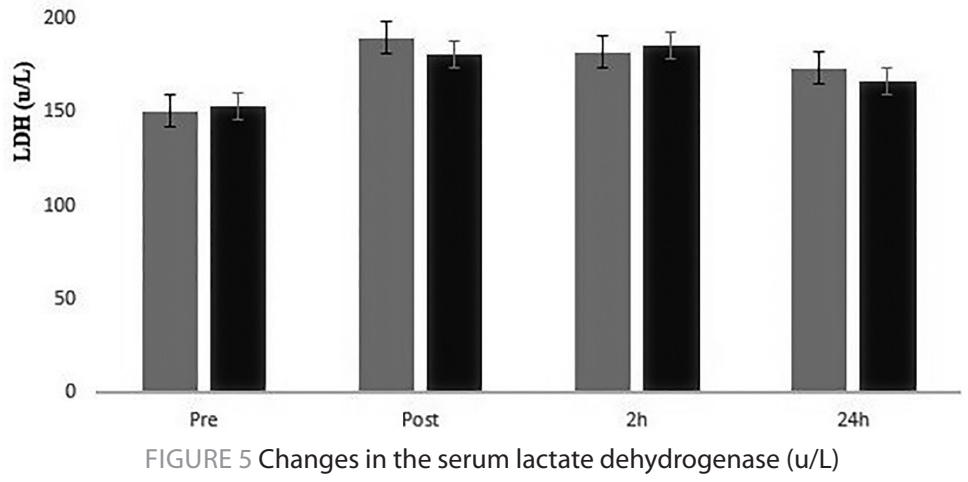




\section{Discussion}

The levels of muscle damage parameters measured in the study show that smoking constitutes a strong risk factor for both cardiac and skeletal damage. In addition to cigarette use, there is a physiologically significant increase in the exercise-induced muscle damage parameters. The results of the study are in agreement with the literature (Maughan et al., 1989; Clarkson et al., 2006; Greer et al., 2007; Lewicki et al., 1987; Coombes et al., 2000; Schumann et al., 2003) and it was noted that the LDH levels measured 2 hours after exercise peaked in both groups. Statistical comparison of the LDH levels between the smoking and non-smoking groups indicated that there was no statistically significant difference between the groups at any time point.

According to our study, in both the smoking and non-smoking groups, there was a significant increase in CK levels immediately after exercise, 2 hours after exercise and 24 hours after exercise compared to the pre-exercise levels. These increases are considered to be due to exercise. The CK level gradually increased in both groups and reached the maximal level 24 hours after exercise. No significant difference was detected in terms of CK levels between the smoker and non-smoker subjects in any of the other periods except 24 hours after exercise. The serum CK level reference interval in a healthy person should be between 45-171 U/L (Schumann et al., 2003) but the CK level of the smoking group was above the normal level even before the exercise. This result showed that the CK levels of the smoking group were higher than the level that a healthy person should have. Another point to note is that the 24-hour CK level of the non-smoking group was lower than the pre-exercise CK value of the smoking group.

Exercise causes excessive disruption of skeletal muscle structure. As a result, $\mathrm{CK}$ is released from the cells (Suzuki et al., 2000). In sedentary individuals, both low and high severity endurance exercises lead to a significant increase in plasma CK levels starting within 6 hours after exercise (Guzel et al., 2007). CK level peaks 6 hours after low-intensity exercise, but it peaks after 24 hours following high-intensity exercise. In another study on untrained individuals, it was observed that with the endurance exercise at the intensity of $70 \%$ of $\mathrm{VO} 2 \mathrm{max}$, the CK level reached peak levels before, after, and even 48 hours after exercise (Coombes \& McNaughton, 2000). In a study examining the effect of smoking on CK isoenzymes, rats were exposed to cigarette smoke for 12 weeks. The serum CK levels of the rats who were exposed to cigarette smoke increased significantly compared to the previous period (Anbarasi et al., 2005). No similar study has been conducted on humans.

Myoglobin is a protein structure found only in the muscles. As a result of muscle or tissue injury, it may get into the blood. Although it is one of the markers of both skeletal and cardiac muscle injury, myoglobin is a parameter that is examined more frequently in the diagnosis of heart attack (Chiu et al., 1999). In a study in which aerobic endurance exercise was performed, myoglobin level during exercise did not show significant increase before exercise, it peaked 1 hour after exercise and returned to normal level 12 hours after exercise (Suzuki et al., 1999).

In a study examining the level of myoglobin according to the condition of the subjects, the subjects were given a gradually increased training for 24 days. According to the obtained data, the myoglobin levels between the first training and the last training decreased significantly (Ross et al., 1983). This situation indicated that the increase in the condition of the subjects was inversely proportional to the level of myoglobin in the blood. Sewright et al. (2008) investigated the effect of gender on eccentric exercise. At the end of the study, no difference was found in serum myoglobin levels between male and female athletes (Sewrigth et al., 2008). In general, with many types of exercise, myoglobin can be said to peak at about 2 hours following exercise (Chiu et al., 1999; Suzuki et al., 1999; Sewright et al., 2008; Volek et al., 2002).

When the effect of endurance exercise on the myoglobin level in the smokers and non-smokers was examined; there was no statistically significant difference between the pre-exercise and post-exercise myoglobin levels in the non-smoker subjects. In the time parameters other than this, no statistically significant difference was detected. In contrast, a comparison of post-exercise and 2 hours after exercise myoglobin levels of untrained smokers showed that the serum myoglobin level 2 hours after exercise was higher. No significant difference other than this was found. The myoglobin levels in both groups peaked 2 hours after exercise. In the comparisons of subjects among smoking and non-smoking groups, there were statistical significances in all periods other than the pre-exercise period. While pre-exercise serum myoglobin levels did not differ, the levels of myoglobin in the blood samples taken immediately after the exercise were observed to be significantly higher in the smoking group. The serum myoglobin levels of the smokers 2 hours after exercise were found to be significantly higher than those of the non-smokers. Also 24 hours after exercise, the myoglobin level in smokers was higher than those of the non-smokers. When the myoglobin levels 24 hours after exercise were examined, it was found that the levels of myoglobin of the non-smokers after 24 hours reduced almost to the baseline level, while it remains at high levels in the smokers. This finding suggested that skeletal muscle and cardiac injury were more prominent in the smokers than non-smokers; furthermore, the recovery time after exercise with the smokers lasted longer than the non-smokers.

The highest reference myoglobin level in a healthy person is known as $90 \mathrm{ng} / \mathrm{ml}$ (De Winter et al., 1995). In the non-smokers, the serum myoglobin level did not exceed the critical value even 2 hours after exercise, while the myoglobin level of the smokers reached $111.14 \mathrm{ng} / \mathrm{mL}$ immediately after exercise. The maximum serum myoglobin level of the smokers reached nearly double the amount of the critical value of $161.28 \mathrm{ng} / \mathrm{ml}$ (Figure 2). The adverse effect of smoking on recovery time and myoglobin level after exercise were observed in this study. 
One of the other determinants of cardiac injury used in this study was troponin-T (De Winter et al., 1995; Lippi et al., 2008; Newby \& Ohman, 2000). Serum troponin level is the most commonly used parameter in case of cardiac damage. There are many studies investigating the increase in serum troponin- $\mathrm{T}$ in relation to various factors. In one study, it was reported that exercise-dependent troponin-T release in adolescent long-distance athletes showed a more significant increase compared to that of adult colleagues (Nie et al., 2011). This may be partially due to the undeveloped antioxidant defence system in the hearts of adolescent athletes (Fu et al., 2009). In another study, remarkable results were detected in the blood samples taken during a marathon. While the troponin-T levels of the athletes gradually increased during the race, they returned to baseline level after exercise. However, they were observed to rise again 4-6 hours after exercise (Middleton et al., 2008). In the literature, no studies investigating the effect of smoking on cardiac troponin level were found.

We determined that troponin-T levels measured after exercise and 2 hours after exercise were not different from baseline levels in the non-smoking group. However, it was noticeable that the troponin-T level 24 hours after exercise was significantly higher than the pre-exercise troponin-T level. In the case of smokers, however, there was a significant difference only between the pre-exercise levels and the levels immediately after exercise. The fact that the pre-exercise basal level did not show a significant increase after exercise indicated that the increase of exercise-dependent cardiac troponin-T level in the smokers was higher than that of the non-smokers. There was a significant difference concerning troponin-T levels between the groups (Figure 1) with regards to pre-exercise and post-exercise measurements. Although the basal levels of troponin-T in non-smokers were higher than those of the smokers, their response to exercise was less pronounced. It was observed that the values of the smoking group did not fall to the basal level 24 hours after exercise while that of the non-smoking group were far below the basal level. When examined broadly, the recovery levels of the non-smokers were more rapid than the smokers.

In the study, the last parameter examined for cardiac injury was CK-MB. It is known that CK-MB increases in the case of cardiac diseases and long-lasting exhausting endurance exercises (Middleton et al., 2008; Newby et al., 2000; Sanchis Gomar et al., 2015; Smith et al., 2004). CK-MB levels may increase and may even rise to 26-fold of its basal levels in exercises that force the metabolism to work extremely hard, such as ultra-marathon (Smith et al., 2004; Siegel et al., 1983). CK-MB levels were found to be higher in rats exposed to cigarette smoke for 12 weeks than those not exposed (Sewrigth et al., 2008). In our study, the pre-exercise levels of both the smoking and non-smoking groups showed a significant increase after exercise (Figure 4). There was no statistically significant difference at all time points between the groups regarding CK-MB levels. The CK$\mathrm{MB}$ levels began to return to the basal level 2 hours after exercise. In this respect, we can say that the CK-MB levels showed almost similar results in both smokers and non-smokers. However, longer exercise may lead to different results.

Since this study was conducted with limited resources, it did not reveal whether there were any changes in the $\mathrm{CK}$ and LDH levels 48 hours after the subjects exercising. Another limitation of the study is that the number of subjects was 17. Different exercise programs that will be performed to the smokers on a higher number of subjects may provide more general and clear results for researchers.

The literature and the results of this study suggest that aerobic exercise causes injury in the skeletal and heart muscles. Except for LDH and CK-MB parameters, it can be stated that long-term use of cigarettes increases the damage caused by exercise in both skeletal and heart muscles and prolongs the recovery time of the smokers more than that of the non-smokers. These results may contribute to the ability of smoking athletes to work and make their recovery period in this direction. It is also believed that the occurrence of cardiac injury due to smoking increases the risk of cardiovascular disease both during exercise and daily life.

In conclusion, when athletes or sedentary people are exposed to cigarette smoke, it affects their performance adversely and increases their level of exhaustion. Thus, they are advised to keep themselves directly or indirectly away from cigarette smoke. In addition to aerobic exercise, high-intensity anaerobic studies in trained smokers and non-smokers should be investigated for future studies. Furthermore, the effects of cigarette smoke exposure while exercising can be examined.

\section{R E F ERE NCES}

Anbarasi, K., Vani, G., Balakrishna, K., \& Devi, C. S. (2005). Creatine kinase isoenzyme patterns upon chronic exposure to cigarette smoke: protective effect of Bacoside A. Vascular pharmacology, 42(2), 57-61. doi: 10.1016/j.vph.2005.01.003

Borg, G.A. (1982). Psychophysical bases of perceived exertion. Med Sci Sports Exerc, 14, 377-381.

Brancaccio, P., Limongelli, F. M., \& Maffulli, N. (2006). Monitoring of serum enzymes in sport. British journal of sports medicine, 40(2), 96-97. doi: 10.1136/bjsm.2005.020719

Brown, L.M., Hill, L. (1991). Some observations on variations in filament overlap in tetanized muscle fibres and fibres stretched during a tetanus, detected in the electron microscope after rapid fixation. J Muscle Res Cell Moti, 12,171-182. doi: 10.1007/BF01774036

Byrne, C, \& Eston, R. (2002). The effect of exercise-induced muscle damage on isometric and dynamic knee extensor strength and vertical jump performance. J Sports Sci, 20, 417-425. doi: 10.1080/026404102317366672 
Chiu, A., Chan, W. K., Cheng, S. H., Leung, C. K., \& Choi, C. H. (1999). Troponin-I, myoglobin, and mass concentration of creatine kinase-MB in acute myocardial infarction. Qjm, 92(12), 711-718. doi: 10.1093/ qjmed/92.12.711

Clarkson, P. M., Kearns, A. K., Rouzier, P., Rubin, R., \& Thompson, P. D. (2006). Serum creatine kinase levels and renal function measures in exertional muscle damage. Medicine \& Science in Sports \& Exercise, 38(4), 623-627. doi: 10.1249/01.mss.0000210192.49210.fc

Colakoglu, F. F., Cayci, B., Yaman, M., Karacan, S., Gonulateş, S., Ipekoglu, G., \& Er, F. (2016). The effects of the intake of an isotonic sports drink before orienteering competitions on skeletal muscle damage. Journal of physical therapy science, 28(11), 3200-3204. doi: 10.1589/jpts.28.3200

Colakoglu, T., Er, F., Ipekoglu, G., Karacan, S., Colakoglu, F. F., \& Zorba, E. (2014). Evaluation of physical, physiological and some performance parameters of the Turkish elite orienteers. Procedia-Social and Behavioral Sciences, 152, 403-408. doi: 10.1016/j.sbspro.2014.09.220

Coombes, J. S., \& McNaughton, L. S. (2000). Effects of branched-chain amino acid supplementation on serum creatine kinase and lactate dehydrogenase after prolonged exercise. Journal of sports medicine and physical fitness, 40(3), 240. doi: 10.1016/j.atherosclerosissup.2018.04.417

De Winter, R. J., Koster, R. W., Sturk, A., \& Sanders, G. T. (1995). Value of myoglobin, troponin T, and CK-MBmass in ruling out an acute myocardial infarction in the emergency room. Circulation, 92(12), 3401-3407. doi: 10.1161/circ.92.12.3401

Ernst, A., \& Zibrak, J. D. (1998). Carbon monoxide poisoning. New England journal of medicine, 339(22), 1603-1608. doi: 10.1056/NEJM199811263392206

Ezzati, M., Henley, S. J., Thun, M. J., \& Lopez, A. D. (2005). Role of smoking in global and regional cardiovascular mortality. Circulation, 112(4),489-497. doi: 10.1161/CIRCULATIONAHA.104.521708

Fox, E. L., Bowers, R. W., \& Foss, M. L. (1993). The physiological basis for exercise and sport (No. Ed. 5). Brown \& Benchmark.

Fu, F., Nie, J., \& Tong, T. K. (2009). Serum cardiac troponin T in adolescent runners: effects of exercise intensity and duration. International journal of sports medicine, 30(03), 168-172. doi: 10.1055/s-0028-1104586

Green, M. S., Jucha, E., \& Luz, Y. (1986). Blood pressure in smokers and nonsmokers: epidemiologic findings. American heart journal, 111(5), 932-940. doi: 10.1016/0002-8703(86)90645-9

Greer, B. K., Woodard, J. L., White, J. P., Arguello, E. M., \& Haymes, E. M. (2007). Branched-chain amino acid supplementation and indicators of muscle damage after endurance exercise. International journal of sport nutrition and exercise metabolism, 17(6), 595-607. doi: 10.1123/ijsnem.17.6.595

Güzel, N. A., Hazar, S., \& Erbas, D. (2007). Effects of different resistance exercise protocols on nitric oxide, lipid peroxidation and creatine kinase activity in sedentary males. Journal of sports science \& medicine, 6(4), 417.

Hachey, B. J., Kontos, M. C., Newby, L. K., Christenson, R. H., Peacock, W. F., Brewer, K. C., \& McCord, J. (2017). Trends in use of biomarker protocols for the evaluation of possible myocardial infarction. Journal of the American Heart Association, 6(9), e005852. doi: 10.1161/JAHA.117.005852

King, C. E., Dodd, S. L., \& Cain, S. M. (1987). O2 delivery to contracting muscle during hypoxic or CO hypoxia. Journal of Applied Physiology, 63(2), 726-732. doi: 10.1152/jappl.1987.63.2.726

Laustiola, K. E., Lassila, R., Kaprio, J., \& Koskenvuo, M. (1988). Decreased beta-adrenergic receptor density and catecholamine response in male cigarette smokers. A study of monozygotic twin pairs discordant for smoking. Circulation, 78(5), 1234-1240. doi: 10.1161/01.CIR.78.5.1234

Lewicki, R., Tchorzewski, H., Denys, A., Kowalska, M., \& Golińska, A. (1987). Effect of physical exercise on some parameters of immunity in conditioned sportsmen. International journal of sports medicine, $8(05)$, 309-314. doi: 10.1055/s-2008-1025675

Lippi, G., Schena, F., Salvagno, G. L., Montagnana, M., Gelati, M., Tarperi, C., ... \& Guidi, G. C. (2008). Influence of a half-marathon run on NT-proBNP and troponin T. Clinical Laboratory Journal For Clinical Laboratories And Laboratories Related, 54(7), 251.

Martinsen, E. W., \& Stephens, T. (1994). Exercise and mental health in clinical and free-living populations. Illinois: Human Kinetics Publishers.

Maughan, R. J., Donnelly, A. E., Gleeson, M., Whiting, P. H., Walker, K. A., \& Clough, P. J. (1989). Delayedonset muscle damage and lipid peroxidation in man after a downhill run. Muscle \& Nerve: Official Journal of the American Association of Electrodiagnostic Medicine, 12(4), 332-336. doi: 10.1002/mus.880120412

Middleton, N., George, K., Whyte, G., Gaze, D., Collinson, P., \& Shave, R. (2008). Cardiac troponin T release is stimulated by endurance exercise in healthy humans. Journal of the American College of Cardiology, 52(22), 1813-1814. doi: 10.1016/j.jacc.2008.03.069

Morton, A. R., \& Holmik, E. V. (1985). The effects of cigarette smoking on maximal oxygen consumption and selected physiological responses of elite team sportsmen. European journal of applied physiology and occupational physiology, 53(4), 348-352. doi: 10.1007/BF00422852

Newby, L. K., \& Ohman, E. M. (2000). The Role of the Troponins and Other Markers of Myocardial. Acute Coronary Syndromes. New York: Marcel Dekker Inc.

Nie, J., Tong, T. K., George, K., Fu, F. H., Lin, H., \& Shi, Q. (2011). Resting and post-exercise serum biomarkers of cardiac and skeletal muscle damage in adolescent runners. Scandinavian journal of medicine \& science in sports, 21(5), 625-629. doi: 10.1111/j.1600-0838.2010.01096.x 
Park, W., Miyachi, M., \& Tanaka, H. (2014). Does aerobic exercise mitigate the effects of cigarette smoking on arterial stiffness?. The Journal of Clinical Hypertension, 16(9), 640-644. doi: 10.1111/jch.12385

Powers, S. K., Lawler, J. O. H. N., Dempsey, J. A., Dodd, S. T. E. P. H. E. N., \& Landry, G. R. E. G. (1989). Effects of incomplete pulmonary gas exchange on VO2 max. Journal of applied physiology, 66(6), 2491-2495. https://doi.org/10.1152/jappl.1989.66.6.2491

Prapavessis, H., Cameron, L., Baldi, J. C., Robinson, S., Borrie, K., Harper, T., \& Grove, J. R. (2007). The effects of exercise and nicotine replacement therapy on smoking rates in women. Addictive behaviors, 32(7), 1416-1432. doi: 10.1016/j.addbeh.2006.10.005

Ross, J. H., Attwood, E. C., Atkin, G. E., \& Villar, R. N. (1983). A study on the effects of severe repetitive exercise on serum myoglobin, creatine kinase, transaminases and lactate dehydrogenase. QJM: An International Journal of Medicine, 52(2), 268-279. doi: 10.1093/oxfordjournals.qjmed.a067760

Sanchis-Gomar, F., Pareja-Galeano, H., Gomez-Cabrera, M. C., Candel, J., Lippi, G., Salvagno, G. L., ... \& Viña, J. (2015). Allopurinol prevents cardiac and skeletal muscle damage in professional soccer players. Scandinavian journal of medicine \& science in sports, 25(1), 110-115. doi: 10.1111/sms.12213

Schumann, G., \& Klauke, R. (2003). New IFCC reference procedures for the determination of catalytic activity concentrations of five enzymes in serum: preliminary upper reference limits obtained in hospitalized subjects. Clinica chimica acta, 327(1-2), 69-79. doi: 10.1016/S0009-8981(02)00341-8

Sewright, K. A., Hubal, M. J., Kearns, A., Holbrook, M. T., \& Clarkson, P. M. (2008). Sex differences in response to maximal eccentric exercise. Medicine and science in sports and exercise, 40(2), 242-251. doi: 10.1249/mss.0b013e31815aedda

Siegel, A.J., Silverman, L.M., Evans, W.J. (1983). Elevated skeletal muscle creatine kinase MB isoenzyme levels in marathon runners. JAMA, 250, 2835-2837. doi: 10.1001/jama.1983.03340200069032

Smith, J. E., Garbutt, G., Lopes, P., \& Pedoe, D. T. (2004). Effects of prolonged strenuous exercise (marathon running) on biochemical and haematological markers used in the investigation of patients in the emergency department. British journal of sports medicine, 38(3), 292-294. doi: 10.1136/bjsm.2002.002873

Srivastava, R., Blackstone, E. H., \& Lauer, M. S. (2000). Association of smoking with abnormal exercise heart rate responses and long-term prognosis in a healthy, population-based cohort. The American journal of medicine, 109(1), 20-26. doi: 10.1016/S0002-9343(00)00441-1

Suzuki, K., Totsuka, M., Nakaji, S., Yamada, M., Kudoh, S., Liu, Q., ... \& Sato, K. (1999). Endurance exercise causes interaction among stress hormones, cytokines, neutrophil dynamics, and muscle damage. Journal of applied physiology, 87(4), 1360-1367. doi: 10.1152/jappl.1999.87.4.1360

Suzuki, K., Yamada, M., Kurakake, S., Okamura, N., Yamaya, K., Liu, Q., ... \& Sugawara, K. (2000). Circulating cytokines and hormones with immunosuppressive but neutrophil-priming potentials rise after endurance exercise in humans. European journal of applied physiology, 81(4), 281-287. doi: 10.1007/s004210050044

Tanaka, H., \& Safar, M. E. (2005). Influence of lifestyle modification on arterial stiffness and wave reflections. AJH, 18, 137-144. doi: 10.1016/j.amjhyper.2004.07.008

Volek, J. S., Kraemer, W. J., Rubin, M. R., Gómez, A. L., Ratamess, N. A., \& Gaynor, P. (2002). L-Carnitine L-tartrate supplementation favorably affects markers of recovery from exercise stress. American Journal of Physiology-Endocrinology and Metabolism, 282(2), 474-482. doi: 10.1152/ajpendo.00277.2001 